Article

\title{
Functional Analysis Reveals the Regulatory Role of PpTST1 Encoding Tonoplast Sugar Transporter in Sugar Accumulation of Peach Fruit
}

\author{
Qian Peng ${ }^{1,2}$, Lu Wang 1,3, Collins Ogutu ${ }^{1,2,4}$, Jingjing Liu ${ }^{1,2}$, Li Liu $^{1,2}$, \\ Md. Dulal Ali Mollah ${ }^{1,2}$ and Yuepeng Han ${ }^{1,3,4, *}$ \\ 1 CAS Key Laboratory of Plant Germplasm Enhancement and Specialty Agriculture, Wuhan Botanical Garden, \\ The Innovative Academy of Seed Design, Chinese Academy of Sciences, Wuhan 430074, China; \\ pengqian114@mails.ucas.ac.cn (Q.P.); wanglu@wbgcas.cn (L.W.); collee52@gmail.com (C.O.); \\ liujingjing18@mails.ucas.ac.cn (J.L.); liuli181@mails.ucas.ac.cn (L.L.); dulal_hort@ru.ac.bd (M.D.A.M.) \\ 2 University of Chinese Academy of Sciences, 19A Yuquanlu, Beijing 100049, China \\ 3 Center of Economic Botany, Core Botanical Gardens, Chinese Academy of Sciences, Wuhan 430074, China \\ 4 Sino-African Joint Research Center, Chinese Academy of Sciences, Wuhan 430074, China \\ * Correspondence: yphan@wbgcas.cn; Tel.: 86-27-8751-0872; Fax: 86-27-8751-0872
}

Received: 17 January 2020; Accepted: 6 February 2020; Published: 7 February 2020

\begin{abstract}
Sugar content is related to fruit sweetness, and the complex mechanisms underlying fruit sugar accumulation still remain elusive. Here, we report a peach PpTST1 gene encoding tonoplast sugar transporter that is located in the quantitative trait loci (QTL) interval on Chr5 controlling fruit sucrose content. One derived Cleaved Amplified Polymorphic Sequence (dCAPS) marker was developed based on a nonsynonymous G/T variant in the third exon of PpTST1. Genotyping of peach cultivars with the dCAPS marker revealed a significant difference in fruit sugar content among genotypes. PpTST1 is located in the tonoplast, and substitution of glutamine by histidine caused by the G/T variation has no impact on subcellular location. The expression profile of PpTST1 exhibited a consistency with the sugar accumulation pattern, and its transient silencing significantly inhibited sugar accumulation in peach fruits. All of these results demonstrated the role of PpTST1 in regulating sugar accumulation in peach fruit. In addition, cis-elements for binding of MYB and WRKY transcript factors were found in the promoter sequence of $P p T S T 1$, suggesting a gene regulatory network of fruit sugar accumulation. Our results are not only helpful for understanding the mechanisms underlying fruit sugar accumulation, but will also be useful for the genetic improvement of fruit sweetness in peach breeding programs.
\end{abstract}

Keywords: Prunus persica; tonoplast sugar transporter; dCAPS; QTL; fruit sweetness

\section{Introduction}

The peach (Prunus persica (L.) Batsch) is considered an important economical fruit crop worldwide. Flavor is one of the key factors affecting fruit quality, and it largely depends on soluble sugar and organic acid contents [1]. Sweetness, determined by the level of soluble sugars, is one of the main attributes that influence the degree of consumer satisfaction for peaches [2]. In mature peach fruit, sucrose accounts for over $54 \%$ of total soluble sugars, which are mainly stored in the vacuole, occupying up to $90 \%$ of the total cell volume [3]. However, the mechanisms underlying sugar accumulation in peach fruit remain largely unknown.

Tonoplast sugar transporters (TSTs) are capable of loading soluble sugars into the vacuole. TSTs belong to a major facilitator superfamily (MFS) that is characterized by 12 transmembrane domains (TMs), with a unique, large central loop [4-6]. The first TST was identified in Arabidopsis; 
it was designated tonoplast monosaccharide transporter (TMT) as its lethal mutation caused a significant decrease in monosaccharide accumulation [7]. However, an increasing number of studies are showing that TMTs function as proton-coupled antiporters that are able to transport sucrose into the vacuole $[8,9]$. Thus, TMTs were renamed as TSTs in a recent study [9].

TSTs have played an important role in sugar accumulation in a variety of crop species. For example, two sorghum TST genes, SbTST1 and SbTST2, show higher levels of expression in the leaf and stem of sweet sorghum, which is rich in soluble sugars when compared to grain sorghum (which mainly accumulates starch) [10]. In rice, OsTMT1 encoding a tonoplast $\mathrm{H}^{+} /$glucose antiporter are highly expressed in vascular tissues, and it is responsible for sugar translocation in both source and sink organs [11]. In Setaria viridis, SvTMTs associated with soluble sugar accumulation are significantly upregulated in the mature zone of the internode [12]. In the sugar beet, a tonoplast sucrose specific transporter, BvTST2.1, is responsible for sucrose accumulation in taproots [9].

Up until now, TSTs have also been identified in many fruit crops, such as the cucumber [13], citrus [14], pear [15,16], apple [17,18], grape [19,20], watermelon [21], and melon [22]. TSTs have proven to be crucial for sugar accumulation in fruit as well. For example, the CITST2 gene is located in a major quantitative trait loci (QTL) for fruit sugar content in watermelon, and its expression shows a well correlation with sugar accumulation in fruit [21]. In Cucumis melo, the CmTST2 gene exhibits higher levels of expression in fruits of high-sugar varieties than in fruits of low-sugar varieties, and its ectopic expression in strawberry and cucumber leads to increased sugar content in fruits [22]. In apples, silencing of MdTMT1 results in a significant decrease in glucose content [17].

During the past three decades, QTLs for fruit sweetness have been identified in all eight chromosomes of the peach [23-29]. However, no candidate genes for fruit sweetness have been identified in the peach. Given that sucrose is the predominant sugar in peach fruit, and TSTs are able to act as $\mathrm{H}^{+}$/sucrose antiporters, we investigated the TST genes in the peach reference genome [30]. Interestingly, a TST gene (Prupe_5G006300), designated PpTST1, is located in a QTL region controlling fruit sucrose content, which accounts for $29 \%$ of phenotypic variation [24,25], and overlaps with the $D$ locus, harboring a major QTL for fruit acidity [25,31]. Here, functional analysis of PpTST1 was carried out and the results demonstrate that it is responsible for sugar accumulation in the peach fruit. In addition, a gene-tagged marker was also developed for PpTST1. Our results are not only helpful for understanding the complex mechanism underlying sugar accumulation in peach fruit, but will also be useful for genetic improvement of fruit sweetness in peach breeding programs.

\section{Results}

2.1. The Expression Profile of PpTST1 Showed a Consistency with the Accumulation Pattern of Soluble Sugars in Peach Fruits

To investigate whether PpTST1 had a potential role in the regulation of sugar accumulation in peach fruit, its expression profile along with sugar accumulation were examined in peach fruit (cvs. Xiahui $6(\mathrm{XH6H})$ and Wanhujing $(\mathrm{WHJ}))$ at different stages of development. As a result, PpTST1 showed an increase in expression levels throughout fruit development, with a peak at the mature stage (120 days after full bloom (DAF)) (Figure 1). Sucrose was the predominant sugar in mature fruits of both $\mathrm{XH} 6 \mathrm{H}$ and $\mathrm{WHJ}$, accounting for $67 \%$ and $77 \%$ of total sugars, respectively. The contents of both sucrose and total sugars showed an increasing trend throughout fruit development. However, the contents of glucose, fructose, and sorbitol showed slight changes throughout fruit development. Overall, the expression profile of $P p T S T 1$ displayed a consistency with the sugar accumulation pattern in peach fruits, suggesting a potential role of PpTST1 in the regulation of fruit sugar accumulation. 

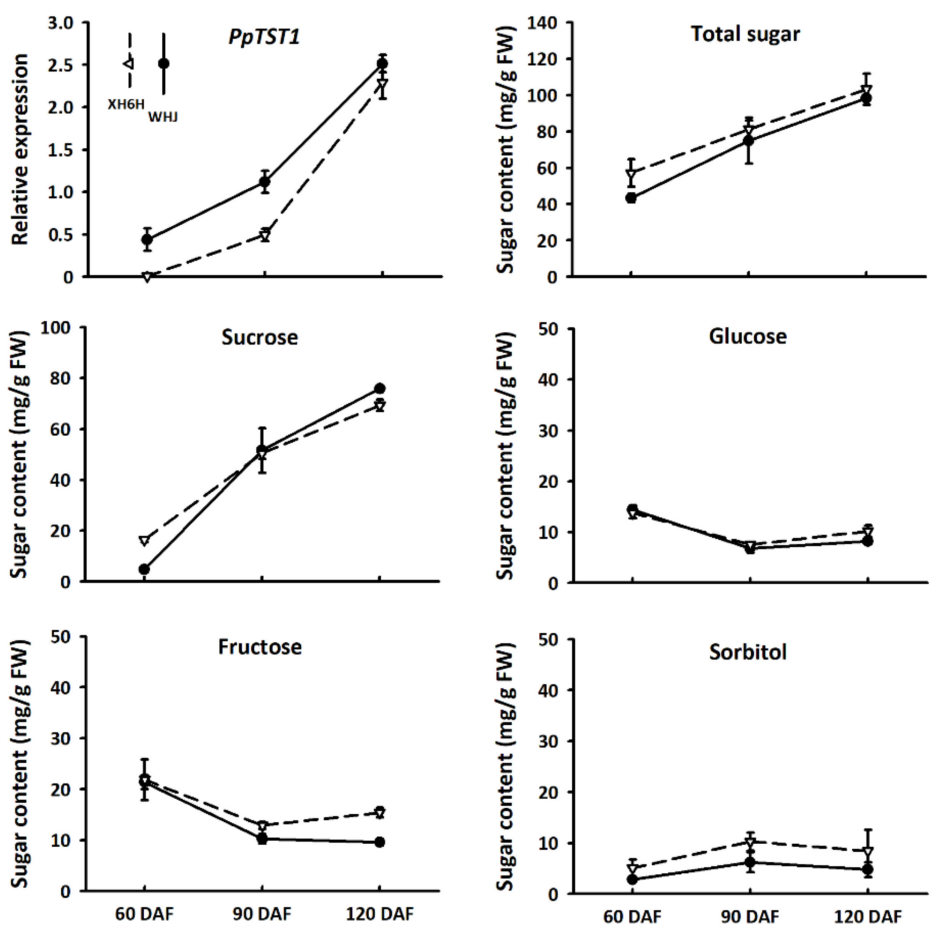

Figure 1. Relative expression of the PpTST1 gene, and accumulation of sugar components in peach fruits at different developmental stages. DAF, days after full bloom. Error bars represent standard error (SE) of three biological replicates.

\subsection{The Impact of PpTST1 on Fruit Sugar Accumulation}

Comparison of our previously reported RNA-Sequencing (Seq) data [32] with the peach reference genome revealed a nonsynonymous G/T single nucleotide polymorphism (SNP) in the third exon of the PpTST1 gene (Figure 2A). To facilitate description, the $\mathrm{G}$ and $\mathrm{T}$ alleles of PpTST1 were designated as PpTST1_G and PpTST1_T, respectively. The G/T SNP was converted into a derived Cleaved Amplified Polymorphic Sequence (dCAPS) marker based on the restriction enzyme PstI. PCR products of the PpTST1_G allele contained PstI recognition sequence CTGCAG , while a corresponding non-symmetrical recognition sequence CTGCAT was generated for PCR products of the PpTST1_T allele. Thus, the PpTST1_G and PpTST1_T alleles could be distinguished by two different PCR fragments, with 184 and $211 \mathrm{bp}$ in size, respectively. This dCAPS marker was subject to screen the G/T polymorphism in a collection of 61 peach cultivars. Accessions with homozygous genotypes, G/G or T/T, had single DNA fragments with 184 or $210 \mathrm{bp}$ in size, respectively, whereas, accessions with the heterozygous genotype G/T generated both the 184-bp and 210-bp DNA fragments (Figure 2B). The majority of tested cultivars $(82 \%)$ belonged to the heterozygous GT genotype, while cultivars with the homozygous G/G or T/T genotypes accounted for $11 \%$ and $7 \%$ of all tested cultivars, respectively.

The sugar content in mature fruits of peach cultivars was measured (Figure 3A). Sucrose was found to be the predominant sugar, and its content ranged from 46.9 to $143.0 \mathrm{mg} / \mathrm{g}$ fresh weight (FW), with an average of $84.6 \mathrm{mg} / \mathrm{g}$ FW. Total sugar content ranged from 122.4 to $241.0 \mathrm{mg} / \mathrm{g} \mathrm{FW}$, with an average of $168.8 \mathrm{mg} / \mathrm{g}$ FW. These results indicated that the contents of sucrose and total sugars showed wide variation among cultivars. By contrast, the content of glucose, fructose, and sorbitol exhibited a small degree of variation. As mentioned above, all of the tested cultivars were divided into three genotypes based on the G/T SNP in the third exon of PpTST1. Comparison of the sugar content between different genotypes revealed that cultivars with either $\mathrm{G} / \mathrm{T}$ or $\mathrm{T} / \mathrm{T}$ genotypes had significantly higher sucrose and total sugar content than cultivars with the G/G genotype (Figure 3B). In contrast, cultivars with either $\mathrm{G} / \mathrm{G}$ or $\mathrm{G} / \mathrm{T}$ genotypes showed significantly higher fructose and glucose contents than cultivars with the $\mathrm{T} / \mathrm{T}$ genotype. However, no significant difference in sorbitol 
content was observed between different genotypes. Taken together, these results suggest that PpTST1 has an impact on fruit sugar accumulation in the peach.

A

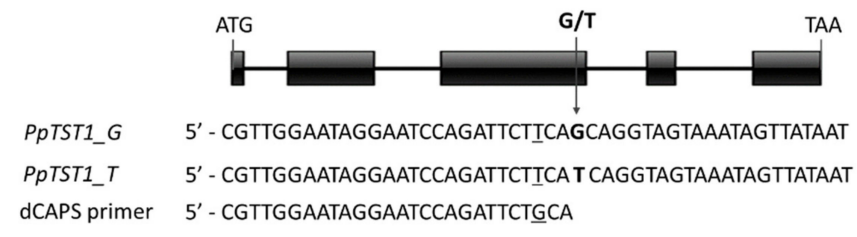

dCAPS primer $\quad 5^{\prime}$ - CGTTGGAATAGGAATCCAGATTCTGGA

$$
\lceil\mathrm{PCR}
$$

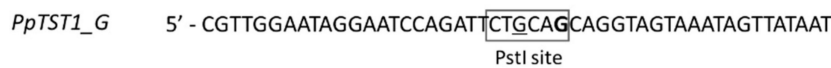

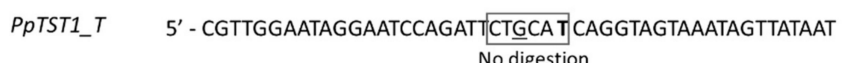

B

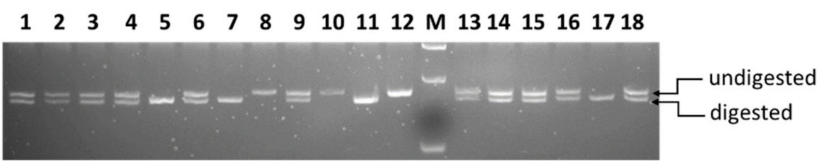

Figure 2. Development and application of gene-tagged molecular marker. (A) derived Cleaved Amplified Polymorphic Sequence (dCAPS) marker development for PpTST1. The G/T single nucleotide polymorphism (SNP) is highlighted in bold. The underlined character indicates the mismatched base for introducing the dCAPS marker. (B) genotyping of peach cultivars with the dCAPS marker. PCR products of each cultivar was digested with PstI and then separated on agarose gel. 1, PANDANA; 2, Diwang 1; 3, Gubatiantao; 4, Hongburuan; 5, 97-1-1; 6, Jinxiayoupantao; 7, Frederica; 8, Hanyin 22; 9 , GHETA; 10, Asama Hakuto; 11, Lianhuang; 12, Zheng 97-4-51; 13, 4-3-3; 14, 99-13-9; 15, Xiahui 6; 16, Zhongyoupantao 2; 17, Meiguowanyou; and 18, Ruiguang 19. M, DNA ladder.

A

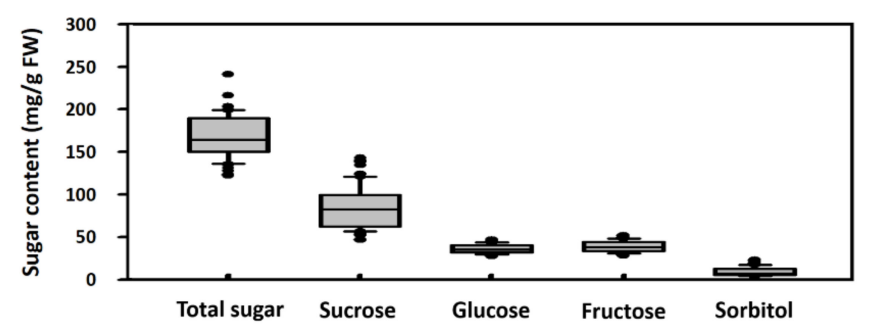

B
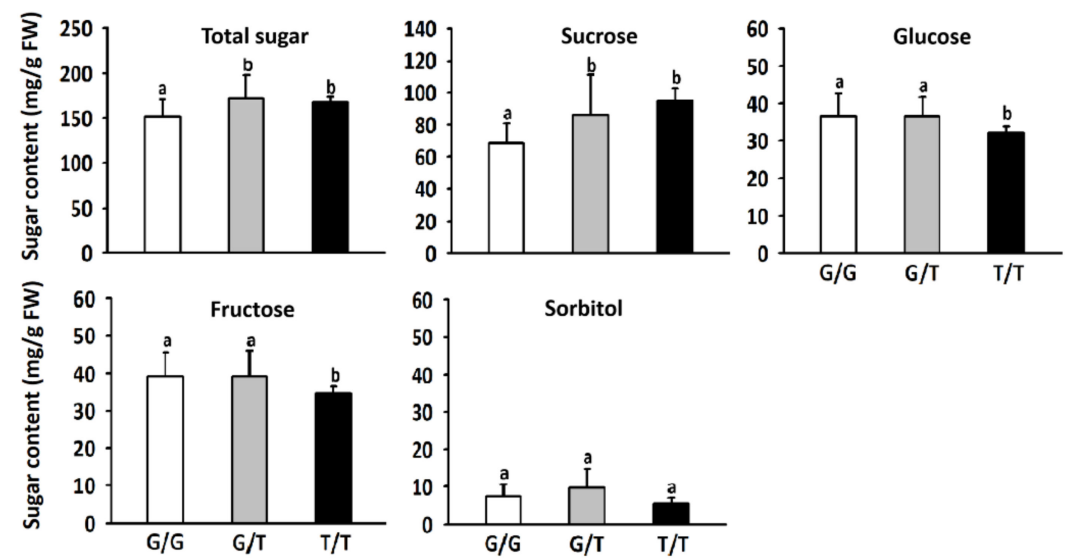

Figure 3. Distribution of soluble sugar content in mature fruits of 61 peach cultivars (A), and mean values of sugar contents in mature peach fruits of different genotypes at the G/T locus (B). Different lowercase letters indicate significant differences between genotypes (Student's $t$ test at $p<0.05$ ). 


\subsection{Assay for Subcelluar Localization of PpTST1 in Tobacco Leaves}

Interestingly, the G/T variant in the third exon of PpTST1 determines a histidine (His) to glutamine (Gln) substitution in the seventh transmembrane domain that was predicted using the Protein Homology/analogY Recognition Engine V 2.0 [33] (Figure S1A,B). To determine whether the His $\rightarrow$ Gln substitution had an effect on subcellular localization, full-length coding sequences of PpTST1_G and PpTST1_T were individually fused with yellow fluorescent protein (YFP) to generate PpTST1_G-YFP and PpTST1_T-YFP constructs under the control driven by the CaMV 35S promoter, respectively. These two constructs were transiently expressed in leaves of Nicotiana benthamiana. Both PpTST1_G-YFP and PpTST1_T-YFP fusion proteins were found to reside in the tonoplast (Figure 4). To clarify the tonoplast location of the proteins from two allele gene, co-localization was conducted using the standard vacuolar membrane marker Vac-rk CD3-975 [34]. The YFP fluorescence of PpTST1-YFP was completely merged with mCherry fluorescence of the tonoplast marker Vac-rk CD3-975 (Figure 4A,B). Altogether, these results showed that two alleles of PpTST1 are both located in the tonoplast, and the G/T SNP in third exon had no effect on subcellular localization.

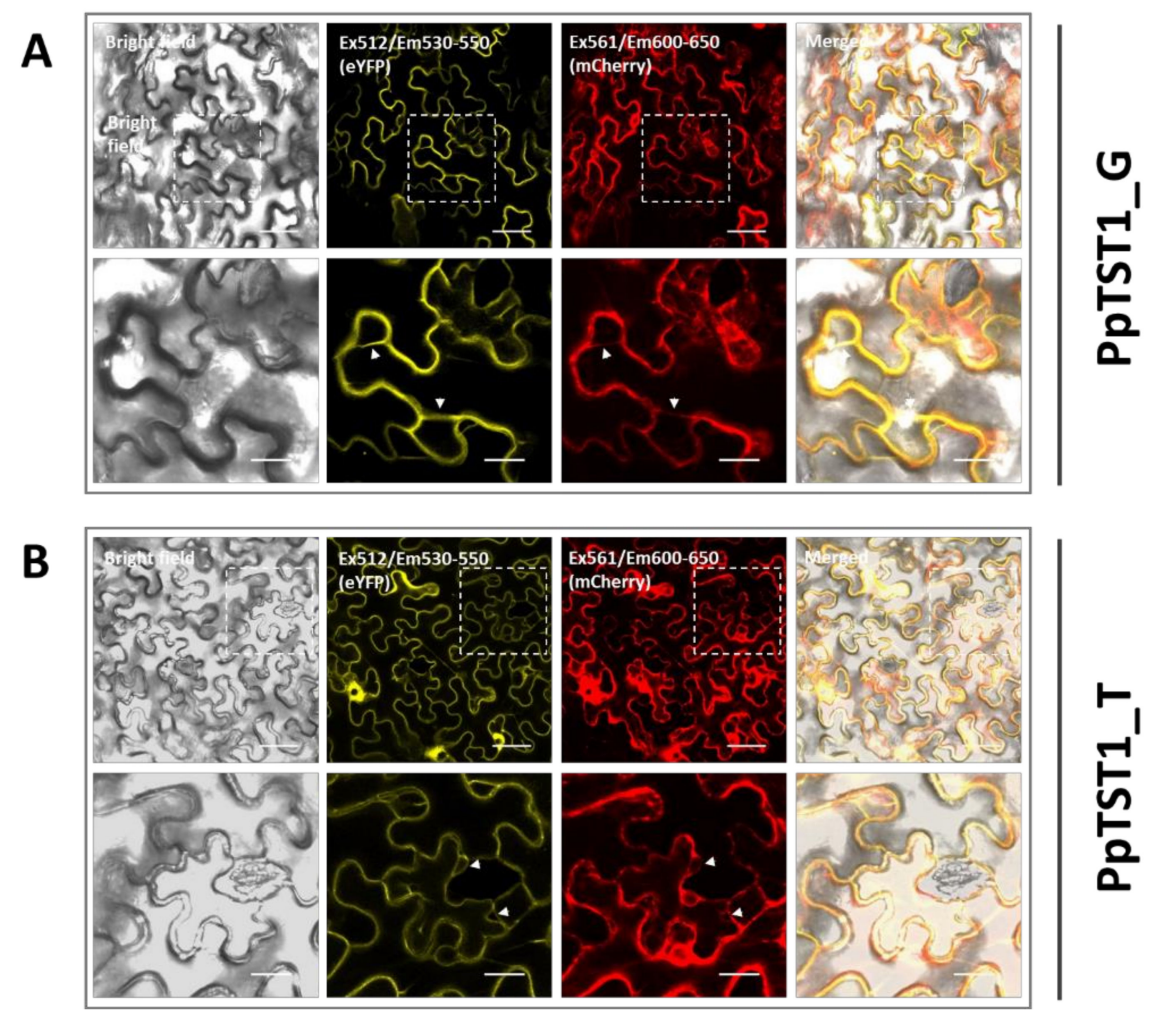

Figure 4. The subcellular localization of PpTST1 in tobacco leaves. Co-expression of pMDC83-eYFPPpTST1_G (A) and pMDC83-eYFP-PpTST1_T (B) with tonoplast marker vac-rk CD3-975 in bright field, YFP channel, mCherry channel, and merged channel, respectively. Bottom lane are the enlarged images of the upper lane. The white arrow indicates vacuole membrane. Upper and bottom lane scale bars are 50 and $20 \mu \mathrm{m}$, respectively.

\subsection{Transient Silencing of PpTST1 Inhibits Sugar Accumulation in Peach Fruit}

The role of PpTST1 in regulating fruit sugar accumulation was also validated using the virus-induced gene silencing (VIGS) system. Specific cDNA sequences covering the end of exon 2 and beginning of exon 3 were amplified and inserted into the pTRV2 vector to generate a pTRV2-PpTST1 construct. Agrobacterium tumefaciens harboring pTRV1 and pTRV2-PpTST1 was injected into fruit at the ripening stage. Real time quantitative PCR (RT-qPCR) analysis indicated that the expression level of PpTST1 was significantly decreased by $50 \%$ in infiltration areas 7 days after transformation with 
pTRV1 and pTRV2-PpTST1, compared with infiltration areas transformed with pTRV1 and pTRV2 (Figure 5A). High performance liquid chromatography (HPLC) analysis further revealed that silencing of PpTST1 reduced the contents of sucrose, glucose, fructose and total sugars by $43.2 \%, 25.0 \%, 23.6 \%$, and $38.1 \%$, respectively. However, no significant decrease was observed for sorbitol content (Figure 5B). Taken together, these results demonstrated that the PpTST1 gene is involved in the regulation of sugar accumulation in peach fruit.

A

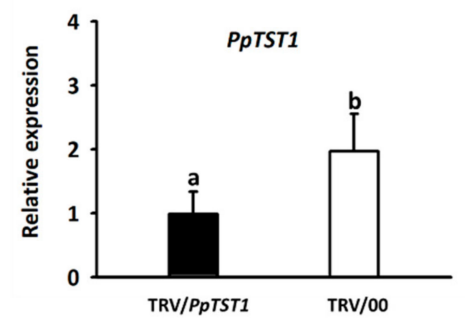

B

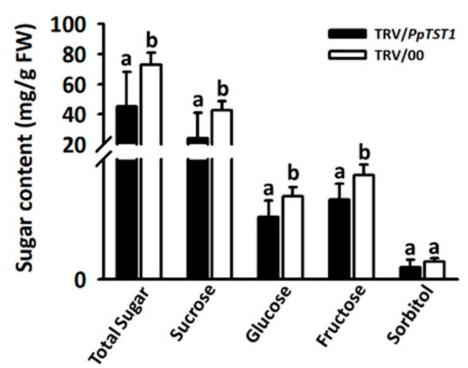

Figure 5. Functional analysis of the PpTST1 gene using the virus-induced gene silencing (VIGS) system in peach fruits. (A) expression of PpTST1 in peach fruit injected with pTRV2-PpTST1/pTRV1 and pTRV2/pTRV1 (control), respectively. (B) sugar accumulation in peach fruit injected with pTRV2-PpTST1/pTRV1 and pTRV2/pTRV1, respectively. Different lowercase letters indicate significant differences between fruits injected with pTRV2-PpTST1/pTRV1 and pTRV2/pTRV1 (Student's $t$ test at $p<0.05)$. pTRV2-PpTST1/pTRV1 and pTRV2/pTRV1 are indicated with TRV/PpTST1 and TRV/00, respectively. Error bars represent standard error (SE) of three biological replicates.

\subsection{Multiple Cis-Elements Are Present in the Promoter Region of PpTST1}

The 2-kb upstream of the start codon of PpTST1 in $\mathrm{XH} 6 \mathrm{H}$ was isolated and annotated using the program PlantCARE [35]. The promoter sequence contained various putative cis-elements involved in response to stress, hormone signaling, and light response (Table S2). In addition, six MYB-related DNA binding motifs and a W-box motif, the highly conserved binding site for WRKY TFs, were also identified. These results indicated that the expression of PpTST1 might be regulated by TFs, such as WRKYs and MYBs, and in response to various environmental stimuli, which is consistent with the finding that fruit sugar accumulation is controlled by environmental and genetic factors $[17,21]$.

\section{Discussion}

Sweetness is one of the most important components of fruit taste quality [1]. Soluble sugars that are primarily stored in the vacuole contribute to the sweetness of fruits [3]. Recent studies show that TSTs are responsible for accumulation of the predominant sugar, sucrose, in fruits or roots of many crops, such as the melon [22], watermelon [21], and sugar beet [9]. Likewise, sucrose also represents the predominant sugar in peach fruit. Up until now, genetic mapping has revealed a total of six QTLs for fruit sucrose content, which are located on chromosomes 1, 3, 5, 6, 7, and 8, respectively [24-27,36,37]. There are four copies of TST genes in the peach genome [38]. Of these four PpTSTs, two, Prupe_7G185700 and Prupe_7G186000, are clustered on Chr7, while the other two, PpTST1 and Prupe_8G180600, are located on Chr5 and Chr8, respectively. The two clustered PpTSTs are located in a QTL interval for sucrose content [26,27], but they show extremely low levels of expression in fruits [38]. PpTST1 is also located within a QTL confidence interval for fruit sucrose content, and its transcript is the most abundant in fruits during the ripening stages compared with other PpTSTs, based on our previous RNA-Seq data [32]. Subcellular localization analysis shows that PpTST1 is located in the vacuolar membrane. Transient silencing of PpTST1 significantly inhibits sucrose accumulation in peach fruit. All of these results suggest that only PpTST1 out of the TST gene family in the peach is likely responsible for sucrose accumulation in fruit, and it is a strong gene candidate in the QTL interval on Chr5 that controls sugar content of peach fruit. 
It is worth noting that silencing of PpTST1 in peach fruit also inhibited hexose accumulation, suggesting that it may also have influence on hexose accumulation. This finding is consistent with previous reports that modification of expression of CmTST2 and CITST2 results in changes in the accumulation of both sucrose and hexose in the melon and watermelon, respectively [21,22]. TSTs function as antiporters that import sugars in exchange for protons, with a 1:1 stoichiometric ratio $[8,39]$. Thus, it is worthy of further study to ascertain the potential impact of the PpTST1 gene on fruit acidity and/or the sugar-acid ratio as it is located in the major $D$ locus for fruit acidity $[25,31]$. In addition, the PpTST gene, Prupe_8G180600, was found to be highly expressed in peach fruit [32,38]. Given that TSTs are capable of loading sucrose and/or hexose into the vacuole [8,39], it is reasonable to speculate that Prupe_8G180600 may be involved in hexose accumulation in peach fruit.

It has been reported that expression levels of TSTs are well consistent with sugar accumulation in many crops $[9,21,22]$. Here, our results show that the expression profile of PpTST1 is also consistent with the fruit sugar accumulation pattern in the peach. These results suggest that the TST genes appear to be regulated primarily at the level of transcription. A nonsynonymous $G / T$ variant in the third exon of $P p T S T 1$, which determines a His to Gln substitution, is located at the seventh transmembrane domain (Figure S1A). However, our study shows that this variant has no impact on subcellular location. Thus, the G/T SNP is unlikely a causal mutation, although its genotypes are related to fruit sugar accumulation. However, it cannot be ruled out that the His to Gln substitution might affect the substrate specificity of PpTST1 as transmembrane domains are known to contribute to sugars translocation across vacuolar membrane [40,41].

Cis-elements, abscisic acid-responsive element (AREB) [17] and $W$ box [21], in the promoter region of TSTs have been reported to play import roles in regulating fruit sugar accumulation. In this study, a variety of putative cis-elements, such as $\mathrm{W}$ box, hormone and light response motifs, and MYB binding sites, are also identified in the promoter sequence of PpTST1. Hence, it is worthy of further study to ascertain whether TFs, such as MYBs and WRKYs, are involved in the regulation of the PpTST1 transcription, which forms a gene regulatory network controlling sugar accumulation in peach fruit.

\section{Materials and Methods}

\subsection{Plant Materials}

Peach (cv. Jinxiu) used for the analysis of tobacco rattle virus (TRV)-mediated virus-induced gene silencing (VIGS) is maintained in the orchard at the Institute of Fruit and Tea, Hubei Academy of Agricultural Sciences, Wuhan, China, while other cultivars are maintained in the peach field at Northwest Agriculture and Forestry University, Yangling, Shaanxi, China. Young leaves were collected in the spring season, and fruit samples were collected at a mature stage. Maturity date was estimated according to previous records, along with fruit that no longer increased in size, softened, easily detached, and showed disappearance of the green skin background color. In addition, two cultivars, Xiahui 6 and Wanhujing, were chosen for gene expression profiling analysis due to their similar duration to maturity. Fruit samples were collected at the following three stages: pit hardening, the second exponential growth, and mature stage, corresponding to 60, 90, and 120 days after full bloom (DAFB), respectively. All fruit samples were peeled and cut into pieces, frozen in liquid nitrogen, and then stored at $-80^{\circ} \mathrm{C}$ until use.

\subsection{DNA Extraction and dCAPS Marker Development}

Genomic DNA was isolated following a previous report [42]. To identify DNA sequence polymorphism in PpTST1, our previously reported RNA-Seq data of peaches [32] were compared with the peach reference genome [30]. A single nucleotide polymorphism (SNP) was detected in the coding region. Subsequently, the web-based free program dCAPS Finder 2.0 [43] was used to convert the SNP into a derived Cleaved Amplified Polymorphic Sequence (dCAPS) marker along with an appropriate restriction enzyme. Primers of the dCAPS marker were designed using online Primer Premier 5 (http://www.premierbiosoft.com/ 
primerdesign/). PCR amplification was conducted in a mixture containing $5 \mu \mathrm{L} 10 \times$ PCR buffer, $4 \mu \mathrm{L}$ $25 \mathrm{mM} \mathrm{MgCl}_{2}, 1 \mu \mathrm{L} 10 \mathrm{mM}$ deoxynucleotide triphosphates (dNTPs), $1 \mu \mathrm{L} 10 \mu \mathrm{M}$ of each forward and reverse primer, $1 \mathrm{U}$ Taq DNA polymerase, and $40 \mathrm{ng}$ DNA template. The PCR program was set as follows: $95{ }^{\circ} \mathrm{C}$ for $5 \mathrm{~min}$; 38 cycles of $95{ }^{\circ} \mathrm{C}$ for $35 \mathrm{~s}, 52{ }^{\circ} \mathrm{C}$ for $35 \mathrm{~s}$ and $72{ }^{\circ} \mathrm{C}$ for $2 \mathrm{~min} 15 \mathrm{~s}$; followed by a final extension at $72{ }^{\circ} \mathrm{C}$ for $30 \mathrm{~min}$. Amplified PCR products were digested using a restriction enzyme (Thermo Fisher Scientific, Waltham, MA, USA) and then separated by electrophoresis on $4 \%$ agarose gel.

\subsection{RNA Isolation and RT-qPCR Analysis}

Total RNA extraction was performed with the RNAprep Pure Plant kit (Tiangen, Beijing, China) according to the manufacturer's instructions, and DNase I (Takara, Dalian, China) was used to remove genomic DNA contamination. Preparation of cDNA templates was carried out with TransScript One-Step gDNA Removal and cDNA Synthesis SuperMix (TRANS, Beijing, China) following the manufacturer's instructions. Real-time quantitative PCR (RT-qPCR) was conducted according to our previously reported protocol [32]. A previously reported actin gene TEF2 [44] was used as a constitutive control, and a negative control for each sample was also performed. Each sample contained three biological replicates. Primer sequences are listed in Table S1.

\subsection{Measurement of Sugar Content and Composition in Peach Fruit}

Fruit samples were ground into powder in liquid nitrogen using an A11 basic Analytical mill (IKA, Darmstadt, Germany). One gram of powder was dissolved in $6 \mathrm{~mL}$ of deionized water. After ultrasonic treatment for $15 \mathrm{~min}$, the mixture was centrifuged at $5000 \times \mathrm{g}$ for $15 \mathrm{~min}$. The supernatants were collected and passed through a filter with a pore size of $0.22 \mu \mathrm{m}$. The filtered supernatants were subject to measure sugar content and composition, with high-performance liquid chromatography (HPLC, Agilent 1260 Infinity, Agilent, Waldbronn, Germany), following our previously reported method [45].

\subsection{Subcellular Localization Analysis of PpTST1 in Tobacco Leaves}

The pMDC83 plasmid used in this study was modified. Briefly, pMDC83 was digested with two restricted enzymes, PmeI and EcoRI, to remove a fragment containing a CaMV 355 promoter, WG Gateway cassette (attR2, ccdB, cmR, attR1 orientation), and green-fluorescent protein (GFP) cassette. Meanwhile, the pFGC-eYFP plasmid was also digested with PmeI and EcoRI, and a small fragment of the T-DNA region containing CaMV $35 S$ promoter, eYFP and OCS terminator was collected. This small fragment was then ligated with the digested pMDC83, resulting in a modified vector, designated pMDC83-eYFP. The entire coding DNA sequence (CDS) without the stop codon of the PpTST1 gene was inserted into pMDC83-eYFP to generate expression construct. The expression vector was transformed into the A. tumefaciens strain GV3101 via the heat shock method and then infiltrated into tobacco leaves. Fluorescence was detected 3 days after infiltration using the confocal microscope (TCS SP8, Leica, Microsystems, Wetzlar, Germany). The sequences of primers used for expression vector construction are listed in Table S1.

\subsection{Functional Analysis of PpTST1 Using the VIGS System in Peach Fruit}

A 350-bp partial cDNA fragment of PpTST1 was amplified and inserted into the EcoRI and BamHI site of the pTRV2 vector with an In-Fusion ${ }^{\circledR}$ HD Cloning Kit (Takara) according to the manufacturer's instructions, resulting in a recombinant construct pTRV2-PpTST1. The pTRV2-PpTST1 vector and two entry vectors, pTRV1 and pTRV2, were individually transferred into the A. tumefaciens strain GV3101 using the heat shock method. Transformants were grown on LB agar plates containing $50 \mathrm{mg} / \mathrm{L}$ rifampicin, $50 \mathrm{mg} / \mathrm{L}$ gentamycin, and $50 \mathrm{mg} / \mathrm{L}$ Kanamycin at $28^{\circ} \mathrm{C}$ for 3 days. Positive Agrobacterium cells were inoculated into LB liquid medium, incubated overnight at $28^{\circ} \mathrm{C}$, and then collected by centrifuging at $4000 \times g$ for $15 \mathrm{~min}$. The pellet was resuspended in infiltration buffer ( $50 \mathrm{mM} \mathrm{MES,} 5 \mathrm{mg} / \mathrm{mL}$ D-glucose, $2 \mathrm{mM} \mathrm{Na}_{3} \mathrm{PO}_{4}, 100 \mu \mathrm{M}$ acetosyringone), and then diluted to an optical density at $600 \mathrm{~nm}$ (OD) $600 \mathrm{~nm}$ of 0.8. Agrobacterium cultures containing pTRV1 were mixed with Agrobacterium cultures containing either 
pTRV2-PpTST1 or pTRV2 in a ratio of 1:1. The infiltration buffer was injected into detached peach fruits at the ripening stage using $1 \mathrm{~mL}$ syringes without the needle. The injected fruits were placed in a growth chamber at $25^{\circ} \mathrm{C}$ under a $16-\mathrm{h}$ light $/ 8$-h dark photoperiod. Peach fruits were subject to RT-qPCR analysis and sugar content measurement 9 days after infiltration. The sequences of primers used for expression vector construction and RT-qPCR are listed in Table S1.

\section{Conclusions}

The PpTST1 gene in the D locus of the peach that encodes a tonoplast sugar transporter, and is a strong candidate that is responsible for fruit sugar accumulation. Further studies are needed to address whether PpTST1 has a potential effect on fruit acidity and/or the sugar-acid ratio, and whether its expression is regulated by TFs, such as MYBs and WRKYs. Our results are helpful for both understanding the mechanisms underlying fruit sugar accumulation and genetic improvement of fruit sweetness in peach breeding programs.

Supplementary Materials: Supplementary Materials can be found at http://www.mdpi.com/1422-0067/21/3/1112/s1.

Author Contributions: Q.P., L.W., and Y.H. designed the experiment. Q.P. and L.W. participated in the dCAPS marker development. Q.P., J.L., and L.L. participated in gene functional studies. Q.P., C.O., and M.D.A.M. analyzed the data. Q.P. wrote the manuscript. C.O., M.D.A.M., and Y.H. revised the manuscript. Y.H. was overall project leader. All authors read and approved the final manuscript.

Funding: This work was supported by grants from the National Key Research and Development Program (2019YFD1000200), the National Natural Science Foundation of China (Grant No. 31872087), and the China Agriculture Research System (grant no. CARS-30).

Conflicts of Interest: The authors declare no conflict of interest.

\section{References}

1. Predieri, S.; Ragazzini, P.; Rondelli, R. Sensory evaluation and peach fruit quality. Acta Hortic. 2006, 713, 429-434. [CrossRef]

2. Delgado, C.; Crisosto, G.M.; Heymann, H.; Crisosto, C.H. Determining the primary drivers of liking to predict consumers' acceptance of fresh nectarines and peaches. J. Food Sci. 2013, 78, S605-S614. [CrossRef] [PubMed]

3. Cantín, C.M.; Gogorcena, Y.; Moreno, M.Á. Analysis of phenotypic variation of sugar profile in different peach and nectarine [Prunus persica (L.) Batsch] breeding progenies. J. Sci. Food Agric. 2009, 89, $1909-1917$. [CrossRef]

4. Reddy, V.S.; Shlykov, M.A.; Castillo, R.; Sun, E.I.; Saier, M.H., Jr. The major facilitator superfamily (MFS) revisited. FEBS J. 2012, 279, 2022-2035. [CrossRef]

5. Yan, N. Structural advances for the major facilitator superfamily (MFS) transporters. Trends Biochem. Sci. 2013, 38, 151-159. [CrossRef]

6. Chen, L.Q.; Cheung, L.S.; Feng, L.; Tanner, W.; Frommer, W.B. Transport of sugars. Annu. Rev. Biochem. 2015, 84, 865-894. [CrossRef]

7. Wormit, A.; Trentmann, O.; Feifer, I.; Lohr, C.; Tjaden, J.; Meyer, S.; Schmidt, U.; Martinoia, E.; Neuhaus, H.E. Molecular identification and physiological characterization of a novel monosaccharide transporter from Arabidopsis involved in vacuolar sugar transport. Plant Cell 2006, 18, 3476-3490. [CrossRef]

8. Schulz, A.; Beyhl, D.; Marten, I.; Wormit, A.; Neuhaus, E.; Poschet, G.; Büttner, M.; Schneider, S.; Sauer, N.; Hedrich, R. Proton-driven sucrose symport and antiport are provided by the vacuolar transporters SUC4 and TMT1/2. Plant J. 2011, 68, 129-136. [CrossRef]

9. Jung, B.; Ludewig, F.; Schulz, A.; Meißner, G.; Wöstefeld, N.; Flügge, U.; Pommerrenig, B.; Wirsching, P.; Sauer, N.; Koch, W.; et al. Identification of the transporter responsible for sucrose accumulation in sugar beet taproots. Nat. Plants 2015, 1, 14001. [CrossRef]

10. Bihmidine, S.; Julius, B.T.; Dweikat, I.; Braun, D.M. Tonoplast Sugar Transporters (SbTSTs) putatively control sucrose accumulation in sweet sorghum stems. Plant Signal. Behav. 2016, 11, e1117721. [CrossRef] 
11. Cho, J.I.; Burla, B.; Lee, D.W.; Ryoo, N.; Hong, S.K.; Kim, H.B.; Eom, J.S.; Choi, S.B.; Cho, M.H.; Bhoo, S.H.; et al. Expression analysis and functional characterization of the monosaccharide transporters, OsTMTs, involving vacuolar sugar transport in rice (Oryza sativa). New Phytol. 2010, 186, 657-668. [CrossRef] [PubMed]

12. Martin, A.P.; Palmer, W.M.; Brown, C.; Abel, C.; Lunn, J.E.; Furbank, R.T.; Grof, C.P.L. A developing Setaria viridis internode: An experimental system for the study of biomass generation in a $\mathrm{C} 4$ model species. Biotechnol. Biofuels 2016, 9, 45. [CrossRef] [PubMed]

13. Hu, B.; Huang, W.F.; Dong, L.L.; Liu, S.Q.; Zhou, Y. Molecular cloning and functional analysis of a sugar transporter gene (CsTST2) from cucumber (Cucumis sativus L.). Biotechnol. Biotec. Eq. 2019, 33, 118-127. [CrossRef]

14. Zheng, Q.M.; Tang, Z.; Xu, Q.; Deng, X.X. Isolation, phylogenetic relationship and expression profiling of sugar transporter genes in sweet orange (Citrus sinensis). Plant Cell Tiss. Org. 2014, 119, 609-624. [CrossRef]

15. Cheng, R.; Cheng, Y.S.; Lü, J.H.; Chen, J.Q.; Wang, Y.Z.; Zhang, S.L.; Zhang, H.P. The gene PbTMT4 from pear (Pyrus bretschneideri) mediates vacuolar sugar transport and strongly affects sugar accumulation in fruit. Physiol. Plantarum 2018, 164, 307-319. [CrossRef]

16. Cheng, R.; Zhang, H.P.; Cheng, Y.S.; Wang, Y.Z.; Wang, G.M.; Zhang, S.L. In silico and expression analysis of the tonoplast monosaccharide transporter (TMT) gene family in Pyrus bretschneideri. J. Hortic. Sci. Biotech. 2018, 93, 366-376. [CrossRef]

17. Ma, Q.J.; Sun, M.H.; Lu, J.; Liu, Y.J.; Hu, D.G.; Hao, Y.J. Transcription factor AREB2 is involved in soluble sugar accumulation by activating sugar transporter and amylase genes. Plant Physiol. 2017, 174, 2348-2362. [CrossRef]

18. Wei, X.Y.; Liu, F.L.; Chen, C.; Ma, F.W.; Li, M.J. The Malus domestica sugar transporter gene family: Identifications based on genome and expression profiling related to the accumulation of fruit sugars. Front. Plant Sci. 2014, 5, 569. [CrossRef]

19. Afoufa-Bastien, D.; Medici, A.; Jeauffre, J.; Coutos-Thévenot, P.; Lemoine, R.; Atanassova, R.; Laloi, M. The Vitis vinifera sugar transporter gene family: Phylogenetic overview and macroarray expression profiling. BMC Plant Biol. 2010, 10, 245. [CrossRef]

20. Çakir, B.; Giachino, R.R.A. VvTMT2 encodes a putative tonoplast monosaccharide transporter expressed during grape berry (Vitis vinifera cv. Sultanine) ripening. Plant Omics 2012, 5, 576.

21. Ren, Y.; Guo, S.G.; Zhang, J.; He, H.J.; Sun, H.H.; Tian, S.W.; Gong, G.Y.; Zhang, H.Y.; Levi, A.; Tadmor, Y.; et al. A tonoplast sugar transporter underlies a sugar accumulation QTL in watermelon. Plant Physiol. 2018, 176, 836-850. [CrossRef]

22. Cheng, J.T.; Wen, S.Y.; Xiao, S.; Lu, B.Y.; Ma, M.R.; Bie, Z.L. Overexpression of the tonoplast sugar transporter CmTST2 in melon fruit increases sugar accumulation. J. Exp. Bot. 2018, 69, 511-523. [CrossRef] [PubMed]

23. Dirlewanger, E.; Graziano, E.; Joobeur, T.; Garriga-Calderé, F.; Cosson, P.; Howad, W.; Arús, P. Comparative mapping and marker-assisted selection in Rosaceae fruit crops. Proc. Natl. Acad. Sci. USA 2004, 101, 9891-9896. [CrossRef] [PubMed]

24. Dirlewanger, E.; Moing, A.; Rothan, C.; Svanella, L.; Pronier, V.; Guye, A.; Plomion, C.; Monet, R. Mapping QTLs controlling fruit quality in peach (Prunus persica (L.) Batsch). Theor. Appl. Genet. 1999, 98, $18-31$. [CrossRef]

25. Etienne, C.; Rothan, C.; Moing, A.; Plomion, C.; Bodénès, C.; Svanella-Dumas, L.; Cosson, P.; Pronier, V.; Monet, R.; Dirlewanger, E. Candidate genes and QTLs for sugar and organic acid content in peach [Prunus persica (L.) Batsch]. Theor. Appl. Genet. 2002, 105, 145-159. [CrossRef] [PubMed]

26. Quilot, B.; Kervella, J.; Génard, M.; Lescourret, F. Analysing the genetic control of peach fruit quality through an ecophysiological model combined with a QTL approach. J. Exp. Bot. 2005, 56, 3083-3092. [CrossRef]

27. Illa, E.; Eduardo, I.; Audergon, J.M.; Barale, F.; Dirlewanger, E.; Li, X.W.; Moing, A.; Lambert, P.; Dantec, L.L.; Gao, Z.S.; et al. Saturating the Prunus (stone fruits) genome with candidate genes for fruit quality. Mol. Breed. 2011, 28, 667-682. [CrossRef]

28. Mora, J.R.H.; Micheletti, D.; Bink, M.; Weg, E.V.D.; Cantín, C.; Nazzicari, N.; Caprera, A.; Dettori, M.T.; Micali, S.; Banchi, E.; et al. Integrated QTL detection for key breeding traits in multiple peach progenies. BMC Genomics 2017, 18, 404.

29. Forcada, C.F.; Guajardo, V.; Chin-Wo, R.S.; Moreno, M.Á. Association mapping analysis for fruit quality traits in Prunus persica using SNP markers. Front. Plant Sci. 2018, 9, 2005. [CrossRef] 
30. International Peach Genome Initiative. The high-quality draft genome of peach (Prunus persica) identifies unique patterns of genetic diversity, domestication and genome evolution. Nat. Genet. 2013, 45, 487-494. [CrossRef]

31. Boudehri, K.; Bendahmane, A.; Cardinet, G.; Troadec, C.; Moing, A.; Dirlewanger, E. Phenotypic and fine genetic characterization of the $D$ locus controlling fruit acidity in peach. BMC Plant Biol. 2009, 19, 59. [CrossRef] [PubMed]

32. Wang, L.; Zhao, S.; Gu, C.; Zhou, Y.; Zhou, H.; Ma, J.J.; Cheng, J.; Han, Y.P. Deep RNA-Seq uncovers the peach transcriptome landscape. Plant Mol. Biol. 2013, 83, 365-377. [CrossRef] [PubMed]

33. Kelley, L.A.; Mezulis, S.; Yates, C.M.; Wass, M.N.; Sternberg, M.J.E. The Phyre2 web portal for protein modeling, prediction and analysis. Nature Protoc. 2015, 10, 845. [CrossRef] [PubMed]

34. Nelson, B.K.; Cai, X.; Nebenführ, A. A multicolored set of in vivo organelle markers for co-localization studies in Arabidopsis and other plants. Plant J. 2007, 51, 1126-1136. [CrossRef]

35. Lescot, M.; Déhais, P.; Thijs, G.; Marchal, K.; Moreau, Y.; Peer, V.P.; Rouzé, P.; Rombauts, S. PlantCARE, a database of plant cis-acting regulatory elements and a portal to tools for in silico analysis of promoter sequences. Nucleic Acids Res. 2002, 30, 325-327. [CrossRef]

36. Cirilli, M.; Bassi, D.; Ciacciulli, A. Sugars in peach fruit: A breeding perspective. Hortic. Res. 2016, 3, 15067. [CrossRef]

37. Desnoues, E.; Baldazzi, V.; Génard, M.; Mauroux, J.B.; Lambert, P.; Confolent, C.; Quilot-Turion, B. Dynamic QTLs for sugars and enzyme activities provide an overview of genetic control of sugar metabolism during peach fruit development. J. Exp. Bot. 2016, 67, 3419-3431. [CrossRef]

38. Vimolmangkang, S.; Zheng, H.; Peng, Q.; Jiang, Q.; Wang, H.L.; Fang, T.; Liao, L.; Wang, L.; He, H.P.; Han, Y.P. Assessment of sugar components and genes involved in the regulation of sucrose accumulation in peach fruit. J. Agric. Food Chem. 2016, 64, 6723-6729. [CrossRef]

39. Wingenter, K.; Schulz, A.; Wormit, A.; Wic, S.; Trentmann, O.; Hoermiller, I.I.; Heyer, A.G.; Marten, I.; Hedrich, R.; Neuhaus, H.E. Increased activity of the vacuolar monosaccharide transporter TMT1 alters cellular sugar partitioning, sugar signaling, and seed yield in Arabidopsis. Plant Physiol. 2010, 154, 665-677. [CrossRef]

40. Diallinas, G. Understanding transporter specificity and the discrete appearance of channel-like gating domains in transporters. Front. Pharmacol. 2014, 5, 207. [CrossRef]

41. Han, L.; Zhu, Y.P.; Liu, M.; Zhou, Y.; Lu, G.Y.; Lan, L.; Wang, X.P.; Zhao, Y.F.; Zhang, C.X.J. Molecular mechanism of substrate recognition and transport by the AtSWEET13 sugar transporter. Proc. Natl. Acad. Sci. USA 2017, 114, 10089-10094. [CrossRef] [PubMed]

42. Kim, C.S.; Lee, C.H.; Shin, J.S.; Chung, Y.S.; Hyung, N.I. A simple and rapid method for isolation of high quality genomic DNA from fruit trees and conifers using PVP. Nucleic Acids Res. 1997, 25, 1085-1086. [CrossRef] [PubMed]

43. Neff, M.M.; Turk, E.; Kalishman, M. Web-based primer design for single nucleotide polymorphism analysis. Trends Genet. 2002, 18, 613-615. [CrossRef]

44. Tong, Z.G.; Gao, Z.H.; Wang, F.; Zhou, J.; Zhang, Z. Selection of reliable reference genes for gene expression studies in peach using real-time PCR. BMC Mol. Biol. 2009, 10, 71. [CrossRef]

45. Zhen, Q.L.; Fang, T.; Peng, Q.; Liao, L.; Zhao, L.; Owiti, A.; Han, Y.P. Developing gene-tagged molecular markers for evaluation of genetic association of apple SWEET genes with fruit sugar accumulation. Hortic. Res. 2018, 5, 14. [CrossRef]

(C) 2020 by the authors. Licensee MDPI, Basel, Switzerland. This article is an open access article distributed under the terms and conditions of the Creative Commons Attribution (CC BY) license (http://creativecommons.org/licenses/by/4.0/). 